\title{
Finite-Precision Implementation of Decoders for LDPC-Coded M-QAM Symstems
}

\author{
Ao Jun ${ }^{1, ~ a, ~ M a ~ C h u n b o ~}{ }^{2, b}$,Cao Guixing Li Cong ${ }^{3, b}$ \\ ${ }^{1}$ College of information and communication, Gulling University of electronic and technology, China \\ Gulling 541004, \\ ${ }^{2}$ China Academy of Space Technology, China Beijing 100094 \\ ajunjunao1@263.net, machunbo@guet.edu.cn,
}

Keywords: LDPC, QAM, quantization, sum-product algorithm, likelihood ration.

\begin{abstract}
In this letter, we address the problem of a finite-precision implementation of demapper and decoder with parity likelihood ration algorithm for LDPC-Coded M-QAM systems. By exploiting the inherent property of the variations in bit reliabilities caused by QAM signal constellation, we introduce a novel method of finite precision representations of decoder messages. This solution is obtained through the adoption of different the number of quantization bits and different quantization law for decoder messages of the different demodulated bit from the same received modulation symbol, that are able to face efficiently the clipping effect. Simulations results demonstrate that for comparable performance the new method can be implemented with much less quantization bits, which can lead to considerably lower decoding cost over the sum-product algorithm for decoding the low density parity check codes.
\end{abstract}

\section{Introduction}

Modern broadcast communications are characterized by increasing throughput requirements. So, there is the need of large spectral efficiencies, which is usually satisfied by employing high order modulation schemes. Higher order modulation schemes, like QAM, put a number of additional problems. In particular, the larger signal-to-noise ratio is required. To solve the problem, attractive solutions is to the adoption of the error correcting codes, e.g. LDPC, which are able to offering remarkable error correction capabilities. In practical hardware implementations of LDPC decoders, bit error rate performance are affected strongly by finite precision representation of the decoder messages. The aim of this paper is to study finite-precision effects on an LDPC coded -QAM system. This topic has been already discussed in previous literature, but most of previous works were limited to consider binary modulation and decoders with sum-product algorithm [4-8]. In [5], the authors show that core hyperbolic functions of the logarithmic Sum Product Algorithm (LLR-SPA) decoder can be effectively implemented through a uniform quantization or a 2-dimation broken line approximation, in the latter case with negligible performance loss. In $[6,7]$, the authors suggest the adoption of 6-bit quantization for the decoder messages as the best trade-off between performance and complexity in coded binary modulation. When considering higher order modulation schemes, more bits are necessary to represent the decoder messages without yielding significant performance degradation [8]. In [9], the authors adopt non-uniform quantization schemes for the decoder messages in LDPC-coded systems using M-QAM schemes. However, the aforementioned references focusing on analyzing finite precision effects of decoder messages with LLR-SPA or its low-complexity versions, like the Min-Sum variant. The subtraction involved in SPA, however, renders it sensitive to the quantization [10].

Here we investigate the performance of the likelihood ration logarithmic quantization, as well as their implementation complexity. Starting with variations in bit reliabilities caused by QAM signal 
constellation, various reduced-complexity variants are derived, in which the reduction in complexity is based on adoption of different the number of quantization bits and different quantization law for decoder messages of the different demodulated bit from the same received modulation symbol.

\section{BIT RELIABLITY LIKELIHOOD RATIO CALCULATION FOR M-QAM}

The most common bit-to-symbol mapping rule for the square 16-QAM signal constellation is the Gray code labeling Fig. 1, whose bit orders is $\left(b_{1}, b_{2}, b_{3}, b_{4}\right)$, and the mappings of the in-phase component $b_{1}, b_{2}$ bits and the quadrature-phase component $b_{3}, b_{4}$ bits on the signal constellation are orthogonal. Therefore, it is sufficient to focus on the in-phase component bits. The same conclusion also applies to the quadrature- phase component bits. For the AWGN channel, the received 16-QAM symbol is $R=S_{m}+\mathrm{N}_{0}$, where $m=1,2 \cdots, 16, R=r_{I}+j r_{Q}$ is received modulation symbol and $N_{0}=n_{I}+j n_{Q}$ is the additive white Gaussian noise, with zero mean, variance $\delta^{2}$.

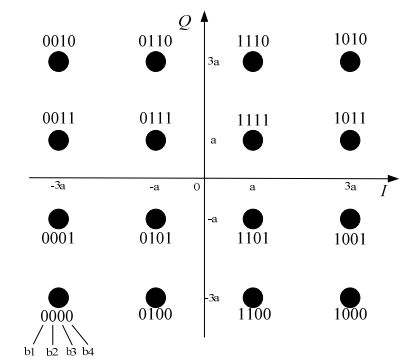

Fig. 1. Gray labeling for 16-QAM

The posteriori likelihood-ratio (LR) which is a soft-metric for the reliability of demodulated bit $b_{k}$ from a received modulation symbol, is defined as follows

$$
\mathrm{LR}_{\mathrm{p}}\left(b_{k}\right)=\frac{\operatorname{Pr}\left\{\mathrm{b}_{t, k}=0 \mid R\right\}}{\operatorname{Pr}\left\{\mathrm{b}_{t, k}=1 \mid R\right\}}
$$

The LRs for the square 16QAM shown in Fig. 1 are:

$$
\begin{aligned}
& \operatorname{LR}_{\mathrm{p}}\left(b_{1}\right)=\frac{e^{-\mathrm{F} \cdot\left(\mathrm{r}_{I}+3 \mathrm{a}\right)^{2}}+e^{-\mathrm{F} \cdot\left(\mathrm{r}_{I}+\mathrm{a}\right)^{2}}}{e^{-\mathrm{F} \cdot\left(\mathrm{r}_{I}-3 \mathrm{a}\right)^{2}}+e^{-\mathrm{F} \cdot\left(\mathrm{r}_{I}-\mathrm{a}\right)^{2}}} \\
& \operatorname{LR}_{\mathrm{p}}\left(b_{3}\right)=\frac{e^{-\mathrm{F} \cdot\left(\mathrm{r}_{\mathrm{p}}+3 \mathrm{a}\right)^{2}}+e^{\left.-\mathrm{F} \cdot \mathrm{r}_{\mathrm{Q}}+\mathrm{at}\right)^{2}}}{e^{-\mathrm{F} \cdot\left(\mathrm{r}_{\mathrm{Q}}-3 \mathrm{aa}\right)^{2}}+e^{\left.-\mathrm{F} \cdot \mathrm{r}_{\mathrm{Q}}-\mathrm{at}\right)^{2}}}
\end{aligned}
$$

$$
\begin{aligned}
\operatorname{LR}_{\mathrm{p}}\left(b_{2}\right) & =\frac{e^{-\mathrm{F} \cdot\left(\mathrm{r}_{\mathrm{I}}-3 \mathrm{aa}\right)^{2}}+e^{-\mathrm{F} \cdot\left(\mathrm{r}_{\mathrm{I}}+3 \mathrm{a}\right)^{2}}}{e^{-\mathrm{F} \cdot\left(\mathrm{r}_{\mathrm{I}}-\mathrm{a}\right)^{2}}+e^{-\mathrm{F} \cdot\left(\mathrm{r}_{\mathrm{I}}+\mathrm{a}\right)^{2}}} \\
\operatorname{LR}_{\mathrm{p}}\left(b_{4}\right) & =\frac{e^{-\mathrm{F} \cdot\left(\mathrm{r}_{\mathrm{I}}-3 \mathrm{aa}\right)^{2}}+e^{-\mathrm{F} \cdot\left(\mathrm{r}_{\mathrm{I}}+3 \mathrm{a}\right)^{2}}}{e^{-\mathrm{F} \cdot\left(\mathrm{r}_{\mathrm{I}}-\mathrm{a}\right)^{2}}+e^{-\mathrm{F} \cdot\left(\mathrm{r}_{\mathrm{I}}+\mathrm{a}\right)^{2}}}
\end{aligned}
$$

Where $F=1 / 2 \delta^{2}$. Under the assumption of the distance between adjacent symbols in Fig. 1 is $2 \mathrm{a}$ $(\mathrm{a}=1)$, the following relationship holds: $\delta^{2}=(M-1) a^{2} / 3 \log _{2} M \cdot r \cdot \mathrm{E}_{b} / \mathrm{N}_{0}$, where $r$ is the code rate. According to equations (2), the average likelihood-ratio logarithm of $b_{1}$ and $b_{2}$ for a Gaussian channel are shown in the plot of Fig. 2 (a), as a function of the average signal-to-noise ratio per bit $E_{b} / N_{0}$.

From the Fig. 2(a) we can see that the average bit reliability of $b_{1}$ is three times as high as $b_{2}$. Higher bit reliability corresponds to higher quantization threshold.

\section{III.Outline of the parity likelihood ration Decoding Algorithm}

The values (2), calculated for all bits of a codeword, are the intrinsic messages given as input to the belief propagation algorithm. They serve to initialize extrinsic messages, which are then updated through the iterated exchange of messages between variable and check nodes in the Tanner graph representing the code. The sum-product algorithm (SPA) and the parity likelihood ration algorithm (PLRA) for LDPC decoding both are based on a factor graph representation of the sparse parity check matrix, but the latter is less sensitive to the quantization effect as it avoids subtraction operation, which is involved in SPA [9].

With each nonzero entry in the parity check matrix $H=\left[h_{1}, h_{2}, \cdots, h_{J}\right]^{T}$ we use the following notations for the messages passing algorithm. Let $v_{i}$ is initial message of variable node $i, V=\left\{V_{j, i}: i=1,2, \cdots, n ; j=1,2, \cdots J\right\}$ be the set of messages out from a variable node $i$ to a check node $j$, and $U=\left\{U_{j, i}: j=1,2, \cdots J ; i=1,2, \cdots, n\right\}$ be the 
output messages from a check node $j$ to a variable node $i$. For 16-QAM, the three steps of one decoding iteration at time $t$ are then as follows:

$$
\begin{gathered}
v_{i}=\operatorname{LR}_{\mathrm{p}}\left(b_{t, k}\right), \quad i=4 t+k \\
U_{j, i}=\operatorname{plr}\left(\mathrm{V}_{j} \backslash \mathrm{V}_{i, j}\right) \\
V_{j, i}=v_{i} \prod_{p \neq j, p=1}^{J} U_{p, i}
\end{gathered}
$$

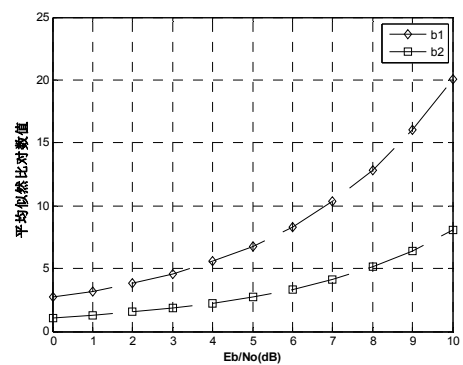

(a) The average bit reliability of demodulated $b_{1}, b_{2}$ bits from a received modulation symbol

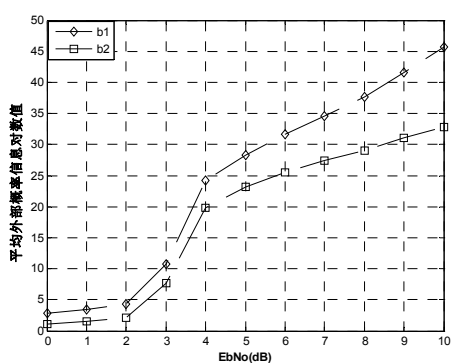

(b) The average bit reliability of $b_{1}, b_{2}$ bits at end of LDPC decoding

Fig. 2. The average LR logarithm for $b_{1}, b_{2}$

\section{QUANTIZATION OF DECODER MESSAGES}

\section{A. Uniform Midtread Quantization}

According to $(2), \operatorname{LR}_{\mathrm{p}}\left(b_{k}\right)$ takes value in $(0, \infty)$.It is thus reasonable to quantize $\operatorname{LR}_{\mathrm{p}}\left(b_{k}\right)$ in the logarithm domain. Let $Q(\ln i)$ represent the quantization levels of decoder message $i$ in the logarithm domain, the input-output characteristic $Q(x)$ of the quantizer is given by the following:

$$
Q_{u}(x)=\left\{\begin{array}{cc}
T & x \geq T \\
\left\lfloor\frac{x}{d}+0.5\right\rfloor \cdot d & -T \leq x \leq T \\
-T & x \leq-T
\end{array}\right.
$$

where $T$ is the saturation threshold, $d$ is the quantization step (dependent on the number of bits $m$ ) and $x$ is the exact values. According to equations (3-5), the average bit reliability of $b_{1}$ and $b_{2}$ bits at end of LDPC decoding are shown in the plot of Fig. 2 (b), in the considered range of signal-to-noise ratios. From the Fig. 2 we can see that PLR decoding algorithm based on message passing and update, resulting in the decoder message increasing compared to the initial behavior, while the message reliability differences still exist between the $b_{1}$ and $b_{2}$ bits. Thus, we resort to adoption of varies the value of quantization bits $n$ and the saturation threshold $T$ according with the bit position. Fig. 2 shows, under the same of signal-to-noise ratios value, that the message reliability of $b_{1}, b_{3}$ are much higher than $b_{2}, b_{4}$. As we expect that the clipping effect has a negative impact on the performance of the decoder, according with these figures, the saturation threshold $T$ for $b_{1}, b_{3}$ should be set larger than that for $b_{2}, b_{4}$, which means the required value of $n$, for $b_{1}$ and $b_{3}$ bits, is higher than that for $b_{2}$ and $b_{4}$ bits. Fig. 3 shows the simulation results of different number of quantization bits and saturation threshold for PLRA for LDPC-coded 16-QAM systems. As comparison, we include the simulated performance for SPA in Fig. 3. Table I lists all the corresponding quantization parameters for Fig. 3 in running simulations. We have adopted PLRA with $n p 1=n p 2=6$ bits uniform quantization, SPA with $n s 1=n s 2=10$ bits uniform quantization. It is seen that without quantization, the last two curves in Fig.3, the performance of PLRA is very close to the SPA. As a general remark, it is possible to observe from these simulations how the PLRA decoder is performing quite well, even when only 6 bits quantization is employed, which further confirm the result in [11], the direct implementation of SPA can be very sensitive to the quantization effect. From Fig. 3, we can see that better performance 
can be achieved by setting $\mathrm{TP} 1=12, \mathrm{TP} 2=6$ compare to the choice of $\mathrm{TP} 1=\mathrm{TP} 2=12$. In our simulation study, the $T$ value is determined empirically (see Fig. 3). It appears that the density evolution scheme can be used to determine the optimum value of the saturation threshold $T$. We are currently looking into this issue for the PLRA technique for LDPC-Coded M-QAM systems.

TABLE I THE CORRESPONDING QUANTIZATION PARAMETERS FOR FIG.
\begin{tabular}{|c|c|c|c|}
\hline quantization parameters & saturation threshold $T$ & quantization bits $n$ \\
\hline \multirow{2}{*}{ PLR } & $\mathrm{b}_{1}, \mathrm{~b}_{3}$ & $\mathrm{~T}_{\mathrm{P} 1}$ & $\mathrm{n}_{\mathrm{P} 1}$ \\
\cline { 2 - 4 } & $\mathrm{b}_{2}, \mathrm{~b}_{4}$ & $\mathrm{~T}_{\mathrm{P} 2}$ & $\mathrm{n}_{\mathrm{P} 2}$ \\
\hline \multirow{2}{*}{$\mathrm{SP}$} & $\mathrm{b}_{1}, \mathrm{~b}_{3}$ & $\mathrm{~T}_{\mathrm{S} 1}$ & $\mathrm{n}_{\mathrm{S} 1}$ \\
\cline { 2 - 4 } & $\mathrm{b}_{2}, \mathrm{~b}_{4}$ & $\mathrm{~T}_{\mathrm{S} 2}$ & $\mathrm{n}_{\mathrm{S} 2}$ \\
\hline
\end{tabular}

\section{B. Non-Uniform Quantization}

From the preceding section, we see that the values of $T_{p 1}$ and $n_{p 1}$, required to obtain the best performance, when employing the quantization characteristic described by (6), can become prohibitively high. Therefore, in this subsection, we provide a non-uniform quantization, that is obtained through a classic compander approach based on uniform midtread quantization, according with (6). Let us define the logarithmic compressor:

$$
\left|x^{\prime}\right|=\log \left(1+\mathrm{K} \cdot\left|x_{T}\right|\right) / \log (1+K)
$$

Where $x_{T}=x / T$ and $x^{\prime}$ are the normalization of the input and output values, respectively. $K$ is a positive real number, that we call the compression "factor". By uniform quantifying $x^{\prime}$ with $T_{T}=1$ and inserting the quantized results $Q_{u}\left(x^{\prime}\right)$ into exponential expandor (7b). Thus, the normalization of the non-uniform quantization output is easily calculated though

$$
x_{\text {nuq }}^{\prime}=\left(\exp \left(\mathrm{I}_{u}\left(x^{\prime}\right) \cdot \log (1+\mathrm{K})\right)-1\right) / \mathrm{K}
$$

Fig. 4 shows the simulation results of decoder messages of $b_{1}, b_{3}$ and $b_{2}, b_{4}$ using different quantization law for PLRA. The curves in Fig.4 marked as "PLR $b_{1}, b_{3}$ quantization law, the number of quantization bits $n_{p 1}-b_{1}, b_{3}$ quantization law, the number of quantization bits $n_{p 2}$ ", where "N" and " $J$ " represents the non-uniform and uniform quantization law, respectively. From Fig. 5, we see that the curve of BER corresponding to the non-uniform quantized version of $n_{p 1}=6$ and uniform quantized version of $n_{p 2}=6$ is very close to the unquantizd one. More specifically, the BER curves relative to the non-uniform quantized version of $n_{p 1}=4$ and uniform quantized version of $n_{p 2}=4$ can achieve slightly better performance than that corresponding to uniform quantization with $n_{p 1}=n_{p 2}=6$, despite the former system adopts a smaller number of quantization bits.

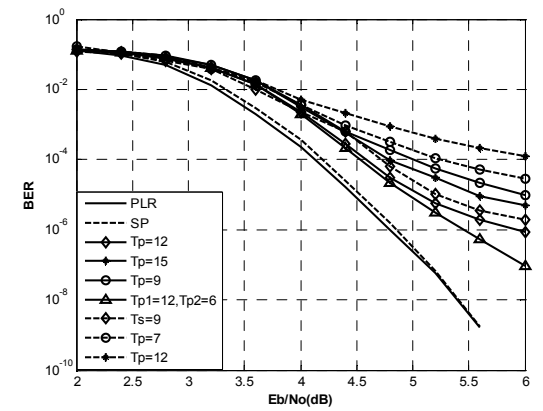

Fig. 3. Performance of the LDPC-Coded M-QAM systems systems

for uniform quantization of decoder messages

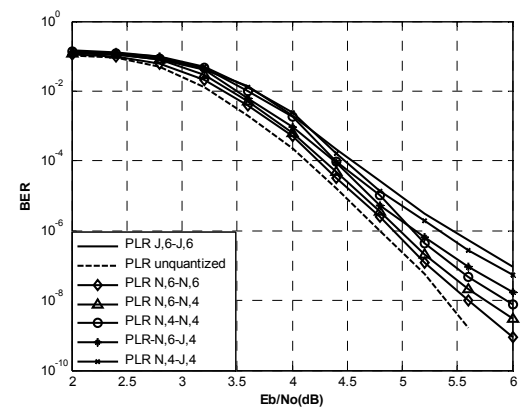

Fig. 4. Performance of the LDPC-Coded M-QAM

for non-uniform quantization of decoder messages

\section{CONCLUSION}

We have addressed the problem of a finite-precision implementation of demapper and decoder with parity likelihood ration algorithm for LDPC-Coded M-QAM systems. The solutions utilize the 
inherent property of the variations in bit reliabilities caused by QAM signal constellation. Simulations results demonstrate that for comparable performance the new method can be implemented with much less quantization bits, which can lead to considerably lower decoding cost with respect to the sum-product algorithm for decoding the low density parity check codes.

\section{Acknowledgements}

This work was financially supported by the National Natural Science Foundation of China (Grant No. 61167006), Key Lab of Cognitive Radio and Information Processing, Ministry of Education (Grant No. 150106)

\section{References}

[1] D. MacKay and R. Neal, Near Shannon limit performance of low density parity check codes, Electronics Letters, 33(6), p. 457-458 (1997).

[2] N. H. Tran and H. H. Nguyen, Signal mappings of 8-ary constellations for bit interleaved coded modulation with iterative decoding, IEEE Trans. Broadcast., 52(1), pp. 92-99, Mar. $2006 .$.

[3] B. Rong, T. Jiang, X. Li, Combine LDPC codes over GF(q) with q-ary modulations for bandwidth efficient transmission, IEEE Trans. Broadcast., 54(1), p. 78-84, (2008).

[4] He Yucheng, Mu Jiangjun, Wang Xinmei, Quantized Decoding of LDPC Codes with Belief Propagation Algorithm, Chinese Journal of Computers, 26(8), p. 934-939, (2003).

[5] M. Shen, H. Niu, H. Liu, and J. Ritcey, Finite precision implementationof LDPC coded M-ary modulation over wireless channels, in Proc. Asilomar Conference on Signals, Systems and Computers, Nov. 2003, vol. 1, p. 114-118.

[6] L. Yang, H. Liu, and C.-J. Richard Shi, Code construction and FPGA implementation of a low-error-floor multi-rate low-density parity-check code decoder, IEEE Trans. Circuits Syst. I, 53(4), p. 892-904,(2006).

[7] S. L. Howard, C. Schlegel, and V. C. Gaudet, Degree-matched check node decoding for regular and irregular LDPCs, IEEE Trans. Circuits Syst. II, 53(10), p. 1054-1058, (2006).

[8] W.Sulek, On the overflow problem in finite precision Turbo decoding message passing, IEEE Trans. Commun., 53(4), p. 1253-1259,(2012).

[9] L. Ping and W. Leung, Decoding low density parity check codes with finite quantization bits, IEEE Commun. Lett., vol. 4, no. 2, pp. 62-64, Feb. 2000.

[10] A. Alexiou, C Bouras, V. Kokkinos, A. Papazois, Modulation and coding scheme selection in multimedia broadcast over a single frequency network-enabled long-term evolution networks, International Journal of Communication Systems, 24(12), p. 1603-1609, (2012). 